\title{
Complexation of DNA with positive spheres: phase diagram of charge inversion and reentrant condensation
}

\author{
Toan T. Nguyen, Boris I. Shklovskii \\ Theoretical Physics Institute, University of Minnesota, 116 Church St. Southeast, Minneapolis, Minnesota 55455
}

October 24, 2018

\begin{abstract}
The phase diagram of a water solution of DNA and oppositely charged spherical macroions is studied. DNA winds around spheres to form beads-on-a-string complexes resembling the chromatin $10 \mathrm{~nm}$ fiber. At small enough concentration of spheres these "artificial chromatin" complexes are negative, while at large enough concentrations of spheres the charge of DNA is inverted by the adsorbed spheres. Charges of complexes stabilize their solutions. In the plane of concentrations of DNA and spheres the phases with positive and negative complexes are separated by another phase, which contains the condensate of neutral DNA-spheres complexes. Thus when the concentration of spheres grows, DNA-spheres complexes experience condensation and resolubilization (or reentrant condensation). Phenomenological theory of the phase diagram of reentrant condensation and charge inversion is suggested. Parameters of this theory are calculated by microscopic theory. It is shown that an important part of the effect of a monovalent salt on the phase diagram can be described by the nontrivial renormalization of the effective linear charge density of DNA wound around a sphere, due to the Onsager-Manning condensation. We argue that our phenomenological phase diagram or reentrant condensation is generic to a large class of strongly asymmetric electrolytes. Possible implication of these results for the natural chromatin are discussed.
\end{abstract}

\section{INTRODUCTION}

In the chromatin a long negative DNA double helix winds around a positive histone octamer to form a complex known as the nucleosomel. Presumably due to their Coulomb repulsion nucleosomes position themselves equidistantly along the DNA helix, forming a periodic necklace or a beads-on-a-string structure, which is also called a $10 \mathrm{~nm}$ fiber. The fact that the self assembly of this structure is very sensitive to the salt concentration shows that electrostatic forces are important. In proper conditions, the $10 \mathrm{~nm}$ fiber self assembles (condenses) into the $30 \mathrm{~nm}$ chromatin fiber which is the major building material of a chromosomel. It is interesting to understand whether electrostatic forces alone are sufficient to form the $10 \mathrm{~nm}$ fiber and determine the range of its stability. This is one of the motivations to study theoretically and experimentally a model of "artificial chromatin", where histone octamers (which themselves are in a complex equilibrium with a population of individual histones) are replaced by identical positive hard spheres (see Fig. 1). In experimental realizations of "arf tificial chromatin", spheres can be colloidal particlestet, micelles 0 or dendrimers . Other motivations are related to many applications of such complexes. One of them is the gene therapy. Inversion of charge of DNA by its complexation with positive spheres should facilitate its contact with a usually negative cell membrane making penetration into the cell more likely. Another application we want to mention here is the possibility of making DNA guided nanowires by complexation of metallic spherical colloids with DNA. When DNA is replaced by synthetic polyelectrolyte (PE), other industrial applications emerge from pharmaceutics to protein extraction and purification.

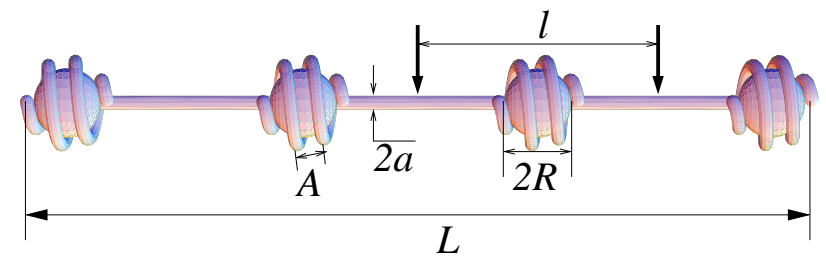

FIG. 1. A beads-on-a-string structure of the complex of a long DNA helix with positive spheres. We assume that the number of turns, $m$, of DNA necessary to neutralize a sphere is large, $m>1$.

In the previous paper 1 we analytically considered a worm-like negative PE (DNA) with the total charge $-Q$ and uniformly charged positive spheres with charge $q$. (Below we use the word DNA instead of PE postponing generalizations to the end of the paper). We studied their complexation in a water solution containing a concentration $p$ of DNA molecules and a concentration $s$ of spheres together with their monovalent counterions and a finite concentration of monovalent salt which provides DebyeHückel screening with the screening radius $r_{s}$. In the Ref. 8 we dealt only with large enough concentrations $s$, neglecting entropy of free spheres. We have shown that, at large $s$, the state of the system is determined by the ratio $s / s_{i}(p)$, where $s_{i}(p)=q / p Q$ is isoelectric concentration at which the total charge of DNA is equal to the total charge of spheres. At $s<s_{i}(p)$ DNA winding around a sphere overscreens it so that the net charge of the sphere becomes negative. Negative spheres repel each other and form a periodic necklace. In the opposite case $s>s_{i}(p)$, more spheres bind to DNA than necessary to neutralize it, thus inverting the charge of DNA. 
The origin of this counterintuitive phenomenon of charge inversion is the correlation induced attraction of a new sphere to a neutral complex: a new sphere approaching a neutral complex pushes away neighboring spheres, unwinds some of DNA from them and winds it on itself. In other words, a new sphere creates an oppositely charged image in the complex and binds to it. It is this additional attraction which leads to charge inversion. In this case, the net charge of each sphere is positive. Positive spheres repel each other and also form a periodic necklace.

Far from the isoelectric point, $s=s_{i}(p)$, complexes have a beads-on-a-string structure (see Fig. 1). They are strongly charged and repel each other in the solution, so that solution is stable. At the isoelectric point, $s=s_{i}(p)$, the sphere net charge and the charge of the whole complex simultaneously cross zero. In the narrow vicinity of the isoelectric point, the charges of complexes (and their Coulomb repulsion) are so small that their short range attraction between complexes due to correlations of solenoids of DNA on different spheres (see Fig. 2) is able to condense them. Complexes form large and weakly charged bundles (see Fig. 3)

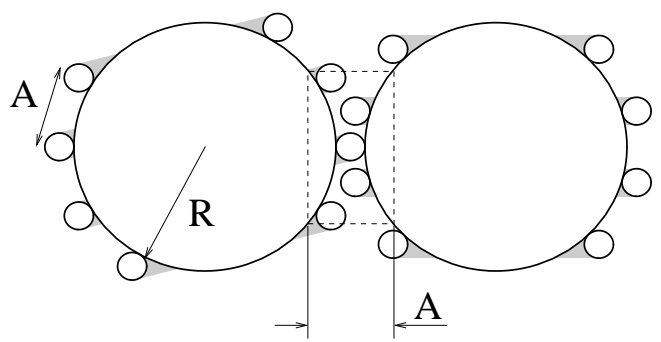

FIG. 2. Cross section through the centers of two touching spheres with worm-like (gray) PE wound around them. At the place where two spheres touch each other (the region bounded by the broken line) the density of PE doubles which in turn leads to a gain in the correlation energy of PE segments wound around the spheres.

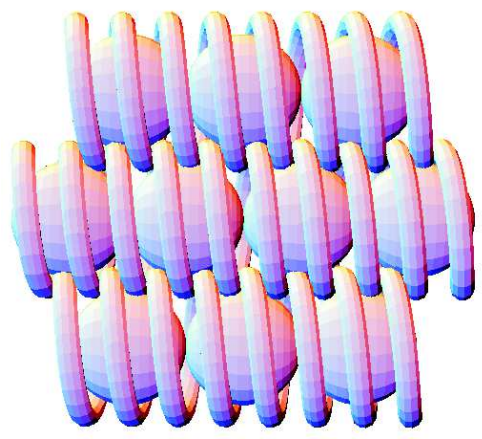

FIG. 3. Almost neutral complexes condense into macroscopic bundles.

At a given concentration $p$ of DNA and growing concentration $s$ of spheres, DNA molecules experience condensation (aggregation) at some critical concentration $s=s_{c}(p)$ below the isoelectric point $s=s_{i}(p)$, and remain in condensed state until another concentration $s=s_{d}(p)$ above the isoelectric point, where the inverted positive charge of the complex becomes so large that condensate dissolves. The electrophoretic mobility changes sign at the isoelectric point $s=s_{i}(p)$ located between $s_{c}(p)$ and $s_{d}(p)$. Inversion of sign of electrophoretic mobility of complexes of DNA with dendrimers was recently reported in Ref. 7 .

In Ref. 8, we dealt only with large $s$ and concentrated mainly on the structure and charge of free necklaces far from the condensation domain. In this paper, we calculate the dependence of the critical concentrations $s_{c}(p)$ and $s_{d}(p)$ for all $(p, s)$ plane and study details of the condensation domain. Our main result is shown by the phase diagram in Fig. A. In this diagram, an aggregate of DNAspheres complexes exists only in the region surrounded by the lines $s_{c}(p)$ and $s_{d}(p)$ (the two solid lines). The domain of large enough concentrations $s$, studied Ref. 8 is shown by gray. In the gray area condensation domain is narrow and encloses isoelectric line $s_{i}(p)$. We call this part of phase diagram the "neck". At smaller $s$ and $p$, the condensation region is found to be much wider so that we call it the "body". The dash line corresponds to the values of $s$ and $p$ at which complexes are neutral. Notice that, in agreement with result of Ref. 8, this line is essentially the isoelectric line for the "neck", but it is high above the isoelectric line for the "body", where substantial fraction of spheres is free.

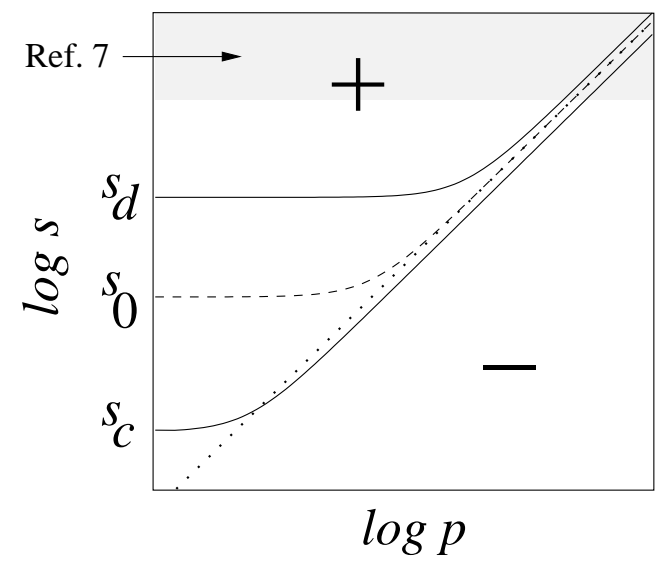

FIG. 4. Phase diagram of a water solution of long DNA molecules and positive spheres in the $(p, s)$ plane. The dotted line corresponds to the isoelectric composition $s=s_{i}(p)$. The dashed line corresponds to the concentration of spheres $s=s_{0}(p)$, where an isolated DNA-spheres complex is neutral. The solid lines $s_{c}(p)$ and $s_{d}(p)$ define the external boundary of the region of existence of macroscopic aggregates. Plus and minus are the signs of the charge of free DNA-sphere complexes and their aggregates above and below the dashed curve. The area of large $s$ studied in previous paper 8 is shown by gray.

The same diagram is shown in linear scale on Fig. 5 . 
At large $s$ and $p$, the phase diagram is extremely simple and centered around the isoelectric line. When we zoom at smaller $s$ and $p$, symmetry with respect to the isoelectric line disappears and we see the fine structure of the condensation domain. There are two light gray regions next to lines $s_{c}(p)$ and $s_{d}(p)$, where condensate coexists with free necklaces. Between them there is the dark gray region were practically all DNA and spheres are consumed by the condensate and concentration of free necklaces is exponentially small. We also study the change in the fraction of free necklaces and their charge when we cross all these regions, for example, by increasing $s$, while keeping $p$ constant (see Fig. 7 below). One of interesting results is that the inversion of the necklace charge happens in a relatively narrow range of $s$.

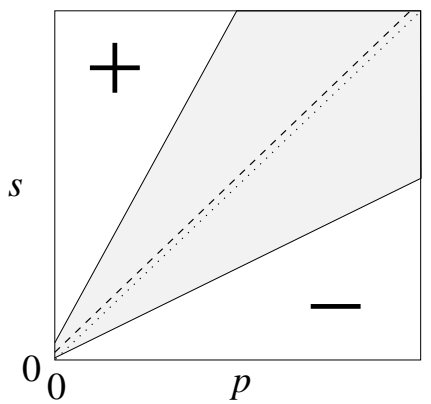

a)

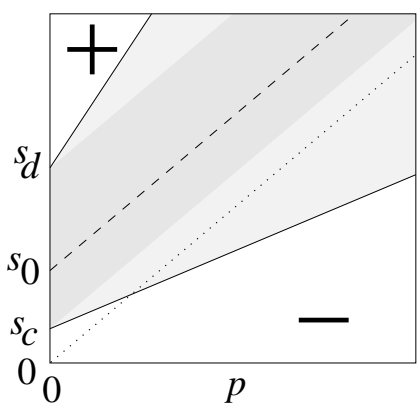

b)
FIG. 5. a) Phase diagram of the mixture of long DNA helix and spheres in linear scale. b) Domain of small $s$ and $p$ is enlarged. On both plots, the dotted and dashed lines and plus and minus signs have the same meaning as in Fig. 4 . The areas of coexistence of aggregates and free complexes are shown by light gray. In the dark gray region, all DNA helices condense. It is shown only in plot b) because it should be very narrow in plot a). One can see that the transitions to and from the complete condensation are abrupt at small $p$ but become less steep with growing $p$.

In Figs. 1 and 0 we did not try to show that the lines $s_{c}(p)$ and $s_{d}(p)$ should merge at extremely small $p$, where translational entropy of DNA molecules becomes important and as a result DNA complexes dissolve. These lines may also merge again when $s$ and $p$ are so large that the fraction of water in the mixture is significantly reduced. Then our phase diagram can also be redrawn as a close loop ternary miscibility diagram.

Bundles of complexes formed in the gray area of phase diagram of Fig. 5 are almost neutral in the sense that their charge is proportional not to the number of aggregated complexes or volume of the bundle, but to the linear size or surface area of bundle. The sign of the charge is however same as for free complexes. It flips on the neutrality line where charge inversion takes place for free complexes.

To derive these phase diagrams, we use two phenomenological parameters. The first one, $\varepsilon$, is the binding energy per sphere of the DNA-spheres complex (neck- lace) in the aggregate. The second one, $s_{0}$, is the concentration of spheres, which is in equilibrium with neutral DNA-spheres complexes. This concentration separates domains of positive and negative complexes at $p=0$. These two phenomenological parameters can be extracted from the experimental phase diagram. They can also be calculated from microscopic theory. In this paper we present comprehensive microscopic theory of these parameters and study the influence of salt on their value. We show below that, generally speaking, screening by salt increases the width of the neck and, at strong screening, increases $s_{0}$ so that the "body" grows at the expense of the "neck".

It is known that the Qnsager-Manning condensation of monovalent counterions 1 renormalizes linear charge density of free DNA helix from the bare value $-\eta$ to critical Onsager-Manning density $-\eta_{c}=-D k_{B} T / e$. Thus one can ask which of the two one should use to calculate charge of DNA molecule, $Q$, and the isoelectric point $s_{i}$. We show that, because of the positive charge of the spheres surface, some DNA counterions are released into solution. As a result, the absolute value of the effective net linear density, $\eta^{*}$, of DNA (which almost neutralizes a sphere) decreases with decreasing screening length $r_{s}$ from the bare density $\eta$ to the Onsager-Manning critical density $\eta_{c}$ according to the formula:

$\eta^{*} \simeq \eta_{c} \frac{\ln \left(r_{s} / a\right)}{\ln \left(A_{0} / 2 \pi a\right)}, \quad\left(a \exp \left(\eta / \eta_{c}\right)>r_{s}>A_{0} / 2 \pi\right)$,

where $A_{0}=4 \pi \eta_{c} R^{2} / Q$ is the distance between DNA turns on the surface of a sphere if $\eta^{*}=\eta_{c}$ and $a$ is the radius of a DNA helix (see Fig. 11). One can show that $\eta^{*}=\eta_{c}$ if $r_{s}<A_{0} / 2 \pi$.

It is the net charge density $\eta^{*}$ of DNA which defines a renormalized isoelectric concentration $s_{i}^{*}(p)=$ $\left(\eta^{*} / \eta\right) s_{i}(p)$. Thus, when $r_{s}$ decreases, the phase diagram moves down together with $s_{i}^{*}(p)$ as shown in Fig. 6. As we mentioned above, screening also leads to some change of the width of condensation domain, but this effect is negligible compared to the shift of the isoelectric line.

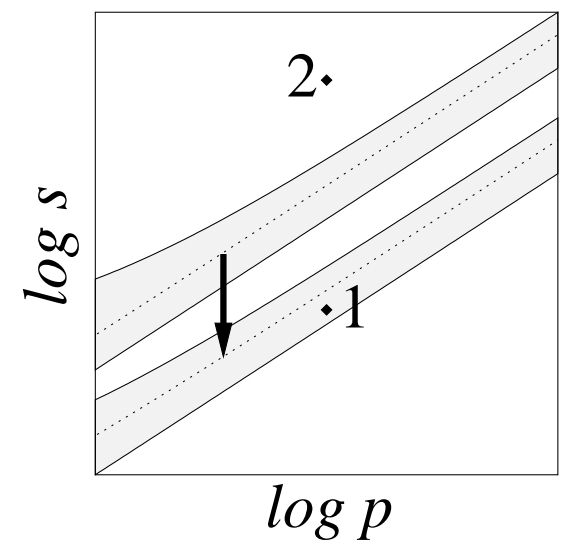

FIG. 6. The shift of isoelectric line and the "neck" of phase diagram (gray) to lower $s$ as a result of the addition of monovalent salt. The points 1 and 2 are used in Sec. IV. 
The phase diagram presented above and its phenomenological theory actually have a much broader applications beyond the complexation of DNA with large spheres. It was actually discovered in experiments of complexation of oppositely charged proteins more than half century ago 1 but have not get a theoretical explanation. Other applications are discussed in Sec. IV. Here, we would like to mention only the well known phenomenon of reentrant condensation of double-helix DNA in solutions with small size multivalent counterions with charge $Z \geq 3$ ( $Z$-ions), such as spermine, a short positive $\mathrm{PE}$ with $Z=4$. At some critical concentration of $Z$-ions, $s_{c}$, DNA abruptly condenses into large bundles 11 . (We use for $Z$-ions the same notation as for spheres to emphasize complete analogy at the phenomenological level). Recently, it was discovered that at another much larger critical $Z$-ion concentration ${ }^{S}$ DNA bundles dissolve again into free DNA helice 1216 . For spermine at very small DNA concentrations, $s_{c}=s_{c}(p \rightarrow 0)=0.025 \mathrm{mM}$ and $s_{d}=s_{d}(p \rightarrow 0)=150 \mathrm{mM}$, if the monovalent salt concentration is not large.

A theory of reentrant condensation of DNA by spermine was given in Ref. 17. It is also based upon two phenomenological parameters $\varepsilon$ and $s_{0}$, which have meaning of the binding energy of DNA per $Z$-ion in the aggregate and the concentration of $Z$-ions which is in equilibrium with neutralized-by- $Z$-ions DNA. By comparison of $s_{c}$ and $s_{d}$ with theory, they were found to be $\varepsilon \simeq 0.3 k_{B} T$ and $s_{0}=3.2 \mathrm{mM}$. Ref. 17, however, dealt only with very small $p$. The phenomenological theory of this paper extends the theory of the spermine induced reentrant condensation to the whole plane $(s, p)$.

Although DNA-spheres complexes and DNA with spermine have the same phase diagram, there are important quantitative differences between them. This is because, as we will see in the next section, the concentration $s_{0}$ decreases exponentially with the binding energy of a $Z$ ion or a sphere with DNA. For large strongly charged spheres, the binding energy of a sphere with wrapping DNA can be so large that $s_{0}$ is extremely small. Therefore, for the case of "artificial chromatin", one most likely deals with the "neck" of the phase diagram of the Fig. Gor, in linear scale, with Fig. 5a. This agrees with the phase diagram obtained in Ref. 18 for DNA-large colloids complexation. On the other hand, for small $Z$-ions, the energy of their binding to DNA is much smaller and $s_{0}$ is an easily observable concentration. In this case, one should see Fig. 5b or the left side ("the body") of the phase diagram of Fig. 1 instead. Indeed the experimental diagram obtained for the spermine 15 looks like the left half of Fig. A.

The paper is organized as follows. In Sec. II, we present a phenomenological theory of the complexation of DNA with spheres. Analytical formulae for the critical concentrations $s_{c}(p)$ and $s_{d}(p)$ are derived and details of the shape of the condensation and decondensation transitions near $s_{c}(p)$ and $s_{d}(p)$ is discussed. In Sec. III we present microscopic theory for parameters $\varepsilon$ and $s_{0}$, for artificial chromatin and study the role of screening by monovalent salt. Onsager-Manning condensation on DNA wrapping around a sphere is considered in Sec. IV. Only in Sec. IV, we return to the discussion of other examples of application of the same phase diagram. In Sec. $\mathrm{V}$, we discuss limits of applicability of our theory of reentrant condensation. In the conclusion, we summarize our results and discuss the possible implication of this theory to the natural chromatin.

\section{PHENOMENOLOGICAL THEORY AND PHASE DIAGRAM OF REENTRANT CONDENSATION}

To begin with, let us first study a complex of a single DNA with $N$ spheres. Its free energy can be written as

$$
f(N)=Q^{* 2} / 2 C+N E(N) .
$$

where $Q^{*}=q N-Q=Q\left(N / N_{i}-1\right)$ is the net charge of the complex ( $q$ and $Q$ are the charges of one sphere and a DNA molecule and $N_{i}=Q / q$ is the number of spheres necessary to neutralize a DNA helix). Except for a specific value of the sphere concentration $s$, the DNAspheres complex is always charged and, therefore, has an extended shape to minimize its Coulomb self-energy. At a length scale larger than the average distance $l$ between the spheres along the complex, the complex can be considered as a charged cylinder with length $L$, radius $l$ (see Fig. 1) and linear charged density $Q^{*} / L$. Then the capacitance $C$ of the complex is:

$$
C=\frac{D L}{2 \ln \left(r_{s} / l+1\right)},
$$

where $D$ is the dielectric constant of water and monovalent salt in solution is treated in the Debye-Hückel approximation with screening length $r_{s}$.

The first term in Eq. (2) is the standard self-energy of a complex with net charge $Q^{*}$ and capacitance $C$. The second term is the correlation energy, which accounts for the discreteness of the spheres charge at the length scale smaller than $l$ (in the beads-on-a-string structure, it is essentially the interaction of a sphere with the DNA coil wound around it). The correlation energy per sphere $E(N)$ is negative and, in general, it is to be calculated from microscopic theory. In this section, it is assumed to be known and is used as an input parameter of the theory. Below, we show that the parameter $s_{0}$ mentioned in the introduction is directly related to $E(N)$.

Given a DNA concentration $p$, we want to calculate the value of the sphere concentration $s_{c}(p)$ and $s_{d}(p)$ where condensation and decondensation of DNA-spheres complexes happen. To do so, let us consider the system in a transition state where aggregates coexist with free complexes. Let $x$ be the fraction of DNA in the aggregates, 
the concentration of free DNA-spheres complexes in solution is then $(1-x) p$. Correspondingly, the concentration of free spheres in solution is $s-x p N_{i}-(1-x) p N$, where $x p N_{i}$ is the concentration of spheres consumed by the neutral macroscopic aggregates, and $(1-x) p N$ is the concentration of spheres bound to the free DNA-spheres complexes in the solution. The free energy per unit volume of the system can then be written as:

$$
\begin{aligned}
& F(N, x ; s, p)=(1-x) p f(N)+x p N_{i}\left[E\left(N_{i}\right)+\varepsilon\right]+ \\
& k_{B} T\left[s-x p N_{i}-(1-x) p N\right] \ln \frac{\left[s-x p N_{i}-(1-x) p N\right] v_{0}}{e} .
\end{aligned}
$$

The first term in Eq. (19) is the free energy density of the free complexes with $f(N)$ given by Eq. (2). The second term is the free energy density of the aggregates, $N_{i} \varepsilon$ is the energy gained per complex by forming the aggregates (compared to a free neutral isolated DNA-spheres complex in solution). This energy gain originates from the correlation-induced short range attraction between complexes mentioned in the introduction ( $\varepsilon$ is negative, see Fig. 2). Like $E(N), \varepsilon$ is to be calculated from microscopic correlation theory, but in this section, it is considered as another input parameter of the theory. The third term in Eq. (4) is the free energy density of the concentration $s-x p N_{i}-(1-x) p N$ of left over free spheres in solution. This concentration is assumed to be small, so that the solution of free spheres is ideal with the normalizing volume $v_{0}$. The translational entropy of DNA is neglected in zeroth order approximation. This is valid if the DNA helix is long enough and the concentration $p$ of DNA is not too small.

For given average concentrations $p$ of DNA and $s$ of spheres, the state of the system can be found by minimizing the free energy (位) with respect to the aggregate fraction $x$ and the number of spheres $N$ bound to a free DNA-spheres complex. This gives:

$$
\begin{aligned}
& k_{B} T \ln \left[\left(s-x p N_{i}-(1-x) p N\right) v_{0}\right]=\mu_{c}(N)+\frac{q Q^{*}}{C} \\
& N_{i} \varepsilon=\frac{\left[Q\left(N / N_{i}-1\right)\right]^{2}}{2 C}+\left(N_{i}-N\right) \times \\
& \quad\left(k_{B} T \ln \left[\left(s-x p N_{i}-(1-x) p N\right) v_{0}\right]-\mu_{c}\left(N_{i}\right)\right) .
\end{aligned}
$$

Eqs. (5) and (6) have very simple physical meanings. Eq. (5) equates the chemical potential of free spheres in solution (the left hand side) and the electrochemical potential of spheres bound to the DNA-spheres complex (the right hand side). Here $Q^{*} / C$ is the average electrostatic potential at the surface of the complex and $\mu_{c}(N)=\partial[N E(N)] / \partial N<0$ is the contribution to the chenpical potential due to sphere correlations in the complex $\mathrm{E}$.

Eq. (6) is the equilibrium condition for neutral complexes. The left hand side of Eq. (6) is the binding energy per complex in the aggregates and the right hand side is the chemical potential of a free DNA-spheres complex in solution. The latter chemical potential is the sum of the complexes' self-energy (neglecting its translational entropy) plus the entropy of $\left(N_{i}-N\right)$ spheres released into solution. (In the dissolved state, a neutral complex releases $\left(N_{i}-N\right)$ spheres charging itself to the charge $\left.Q\left(N / N_{i}-1\right).\right)$

Before progressing further, it should be mentioned that, both lengths $L$ and $l$ are, generally speaking, functions of the number of spheres in the complex, $N$. A detail calculation on the dependence of $L$ and $l$ on $N$ is given in Ref. 8, where the structure of complexes far from isoelectric point (deep in the plus and minus region of the phase diagram of Fig. (1) was considered. In this paper, however, we concentrate on the phase diagram of the reentrant condensation. In the vicinity of the condensation region, the complexes are almost neutral. Therefore, in the above minimization of the free energy, $L$ and $l$ (and hence the capacitance $C$ ) are considered as independent of $N$ and equal to their value for a neutral complex. Indeed, if we consider explicitly the dependence of $L, l$ and $C$ on $N$, on the right hand side of Eq. (5) we get an additional term of the order $Q^{*} / Q$ compared to the second term. It is shown below that $Q^{*} / Q$ is proportional to the square root of the ratio

$$
|\varepsilon C / q Q| \ll 1,
$$

where $\varepsilon$ is the condensate binding energy per sphere and $q Q / C$ is the interaction energy of a sphere with the whole DNA molecule. Thus, the dependence of $L, l$ and $C$ on $N$ can be ignored. The inequality (7) is justified because, as shown in Fig. 2, $\varepsilon$ involves only interactions with a small part of DNA molecule winding around a spheres. A more quantitative justification of this assumption is given in the next section where microscopic theory of DNA-spheres interactions in the complexes and the condensate is given.

If we define a sphere concentration $s_{0}$ as

$$
s_{0}=\exp \left(-\left|\mu_{c}\left(N_{i}\right)\right| / k_{B} T\right) / v_{0},
$$

Eq. (5) can be rewritten as

$$
k_{B} T \ln \frac{s-x p N_{i}-(1-x) p N}{s_{0}}=\left(\frac{N}{N_{i}}-1\right) \frac{q Q}{C} .
$$

From this equation, one can see that, when the concentration of free spheres, $s-x p N_{i}-(1-x) p N$, is greater than the concentration $s_{0}, N / N_{i}-1$ is positive indicating a charge inversion effect. Because Coulomb interaction between spheres is much larger than thermal energy, $\left|\mu_{c}(N)\right| \gg k_{B} T, s_{0}$ is an exponentially small concentration. The range of $s$ where DNA-spheres complexes are overcharged, therefore, is easily accessible experimentally. One also sees that, without correlations, $\mu_{c}=0$, $s_{0}=1 / v_{0}$ and charge inversion can never be observed. This confirms that correlation is the driving force for charge inversion. 
Using Eq. (5) and the assumption that the charge of a complex near condensation region is almost zero so that $\mu_{c}(N) \simeq \mu_{c}\left(N_{i}\right)$, we can rewrite Eq. (6) as

$$
|\varepsilon|=\left(\frac{N}{N_{i}}-1\right)^{2} \frac{q Q}{2 C} .
$$

Equations (9) and (10) make up the core of our theory. They contain only two phenomenological parameters $\varepsilon$ and $s_{0}$. The former describes the strength of short range attraction between complexes and the later describes the strength of sphere-DNA binding, both depend on the charge and size of spheres. Knowing them, one can easily calculate the fraction $x$ of DNA in the condensate and the net charge $Q^{*}=Q\left(N / N_{i}-1\right)$ of free complexes as functions of $s$ and $p$. Thus, the whole phase diagram of the reentrant condensation can be constructed.

Let us consider the limit of small DNA concentration $p$. In this case, neglecting $p$ inside the logarithmic functions, we can rewrite Eqs. (9) and (10) as

$$
\begin{aligned}
& k_{B} T \ln \frac{s}{s_{0}}=\left(\frac{N}{N_{i}}-1\right) \frac{q Q}{C}, \\
& \frac{|\varepsilon|}{k_{B} T}=\frac{k_{B} T C}{2 q Q} \ln ^{2} \frac{s}{s_{0}} .
\end{aligned}
$$

Obviously, there are two solutions $s_{c}$ and $s_{d}$ for Eq. 12):

$$
s_{c, d}=s_{0} \exp \left(\mp \frac{1}{k_{B} T} \sqrt{|\varepsilon| \frac{2 q Q}{C}}\right) .
$$

The solution with the minus sign, $s_{c}<s_{0}$, corresponds to the concentration of spheres at which DNA complexes start to condense forming aggregates. The other solution with the plus sign, $s_{d}>s_{0}$, corresponds to the concentration of spheres at which the aggregates dissolve again into free DNA-spheres complexes in solution. In Fig. 4 and Fig. $5 \mathrm{~b}$, the concentrations $s_{0}, s_{c}$ and $s_{d}$ are shown on the vertical axis.

The meaning of the concentration $s_{0}$ is also transparent in this $p \rightarrow 0$ limit. It corresponds to the sphere concentration at which a free complex is neutral $\left(N=N_{i}\right)$.

Let us now study the dependence of the threshold condensation and decondensation concentrations $s_{c}(p)$ and $s_{d}(p)$ on the DNA concentration $p$. Because the binding energy $\varepsilon$ is a constant, Eq. (10) shows that, in the regime of coexistence of aggregates and free complexes, $N$ is a constant with respect to $p$ and $s$. Let us denote this constant by $N_{c}$ for the condensation transition and by $N_{d}$ for the decondensation transition:

$$
N_{c, d}=N_{i}(1 \mp \sqrt{2|\varepsilon| C / q Q})
$$

The deviations of $N_{c}$ and $N_{d}$ from $N_{i}$ is related to the effective charges of the DNA-spheres complexes when they are in equilibrium with their aggregates:

$$
Q_{c, d}^{*}=Q\left(N_{c, d} / N_{i}-1\right)=\mp Q \sqrt{2|\varepsilon| C / q Q} .
$$

Together with the assumed inequality (7), Eq. (15) confirms that the net charge of a complex near condensation region is very small compare to the bare charge of the DNA.

Eq. (9), in turn, leads to the conclusion that in the coexistence regime, the concentration of free spheres $\left[s-x p N_{i}-(1-x) p N_{c, d}\right]$ in solution is a constant independent of $p$. These constants, of course, are equal to the concentrations $s_{c}$ and $s_{d}$ obtained above for the limit $p \rightarrow 0$. Thus, for the condensation transition,

$$
s(p ; x)-x p N_{i}-(1-x) p N_{c}=s_{c},
$$

and correspondingly, for the decondensation transition

$$
s(p ; x)-x p N_{i}-(1-x) p N_{d}=s_{d} .
$$

Now one can easily find the threshold concentrations $s_{c}(p)$ (where DNA-spheres complexes start to condense into bundles) and $s_{d}(p)$ (where all the bundles dissolve again). Putting $x=0$ in Eqs. (16) and (17) one gets:

$$
\begin{aligned}
& s_{c}(p)=s_{c}+p N_{c}, \\
& s_{d}(p)=s_{d}+p N_{d} .
\end{aligned}
$$

Thus we have simple linear expressions for the condensation and decondensation threshold sphere concentrations as functions of the DNA concentration. These functions are plotted in Fig. 5 by the solid lines. The same phase diagram is plotted in log-log scale in Fig. 4 where the lines distinguishing the coexistence and the complete condensation regions are omitted for clarity.

Putting $x=1$ in Eqs. (16) and (17), one can easily calculate the two sphere concentrations between which the aggregates consume all the DNA-spheres complexes (we are talking about very long DNA neglecting its translational entropy):

$$
\begin{aligned}
& s_{c}^{\prime}(p)=s_{c}(p ; x=1)=s_{c}+p N_{i}, \\
& s_{d}^{\prime}(p)=s_{d}(p ; x=1)=s_{d}+p N_{i} .
\end{aligned}
$$

One concludes that the width of the range of concentration $s$ where $100 \%$ DNA is aggregated, $s_{d}^{\prime}(p)-s_{c}^{\prime}(p)=$ $s_{d}-s_{c}$, is a constant. Remarkably, this region actually does not enclose the isoelectric line $s_{i}=p N_{i}$. In Fig. 5b, the complete condensation region is shaded dark gray.

The sphere concentration $s_{0}(p)$ at which a free complex is neutral can also be calculated in this model. Setting $N=N_{i}$ in Eq. (9) gives

$$
s_{0}(p)=s_{0}+p N_{i}
$$

As expected, $s_{0}(p)$ lies in between $s_{c}^{\prime}(p)$ and $s_{d}^{\prime}(p)$ (compare Eq. (22) with Eqs. (20) and (21)). In the phase diagrams of Fig. 1 and 5 , the concentration $s_{0}(p)$ is plotted by the dash line. At large enough $p$, this concentration 
is close to the isoelectric point. At small $p$, however, it saturates at the finite concentration $s_{0}$.

It is useful to study the relative width of the condensation region:

$$
\frac{\Delta s(p)}{s_{0}(p)}=\frac{s_{d}(p)-s_{c}(p)}{s_{0}(p)}=\frac{s_{d}-s_{c}+p\left(N_{d}-N_{c}\right)}{s_{0}+p N_{i}} .
$$

At small $p$

$$
\frac{\Delta s(p)}{s_{0}(p)}=\frac{s_{d}-s_{c}}{s_{0}}=2 \sinh \left(\frac{1}{k_{B} T} \sqrt{|\varepsilon| \frac{2 q Q}{C}}\right) .
$$

For large spheres, Coulomb interactions are much larger than the thermal energy, the argument of sinh function is large, so that this relative width is exponentially large. It is shown as the "body" of the phase diagram (Fig. 迆). On the other hand at large $p$

$$
\frac{\Delta s(p)}{s_{0}(p)}=\frac{N_{d}-N_{c}}{N_{i}}=\sqrt{\frac{8|\varepsilon| C}{q Q}} \ll 1 .
$$

The narrowing of relative width $\Delta s(p) / s_{0}(p)$ with growing $p$ from the "body" to the "neck" of the phase diagram is clearly seen in the log-log scale of the phase diagram (Fig. 4). (Note that the "neck" encloses the isoelectric line as Eqs. (18) and (19) suggest.) The width of the condensation region, of course, always increases with $p$ as shown in Fig. 5.

We would like to note that, because of the exponential dependence of the concentration $s_{0}$ on the correlation chemical potential of spheres $\mu_{c}$ (see Eq. (8)), the accessible range of the diagram is very sensitive to the size and charge of spheres. When spheres are large and highly charged, $\left|\mu_{c}\right| / k_{B} T$ is very large, $s_{0}$ is unrealistically small and one can access only the right side of this phase diagram (the "neck"). For spheres of smaller size $s_{0}$ may be a more reasonable concentration and one can see also the left half of the phase diagram ("the body").

Given the sphere and DNA concentrations, $s$ and $p$, one can also calculate the fraction $(1-x)$ of DNA dissolved in the solution.

$$
1-x(s)= \begin{cases}1 & s<s_{c}(p), \\ \frac{-s+s_{c}+s_{i}}{s_{i}\left(1-N_{c} / N_{i}\right)} & s_{d}^{\prime}(p)<s<s_{d}(p), \\ 0 & s_{c}^{\prime}(p)<s<s_{d}^{\prime}(p), \\ \frac{s-s_{d}-s_{i}}{s_{i}\left(N_{d} / N_{i}-1\right)} & s_{d}^{\prime}(p)<s<s_{d}(p), \\ 1 & s>s_{d}(p) .\end{cases}
$$

This result is plotted in Fig. 7 by the solid line. The slopes of $x(s)$ in the condensation coexistence region and in the decondensation coexistence region are equal in magnitude. They are inversely proportional to the DNA concentration $p$. Therefore, at small $p$, the transitions from $x=0$ to $x=1$ and vice versa are very abrupt. At large $p$, the coexistence region is wider. This fact is shown in the phase diagram of Fig. 5. The widths of the light gray regions corresponding to coexistence are zero at $p \rightarrow 0$ and increase linearly with $p$. In the limit of large $p$, the function $1-x(s)$ acquires a $\mathrm{V}$-shape form between $s_{c}(p)$ and $s_{d}(p)$.

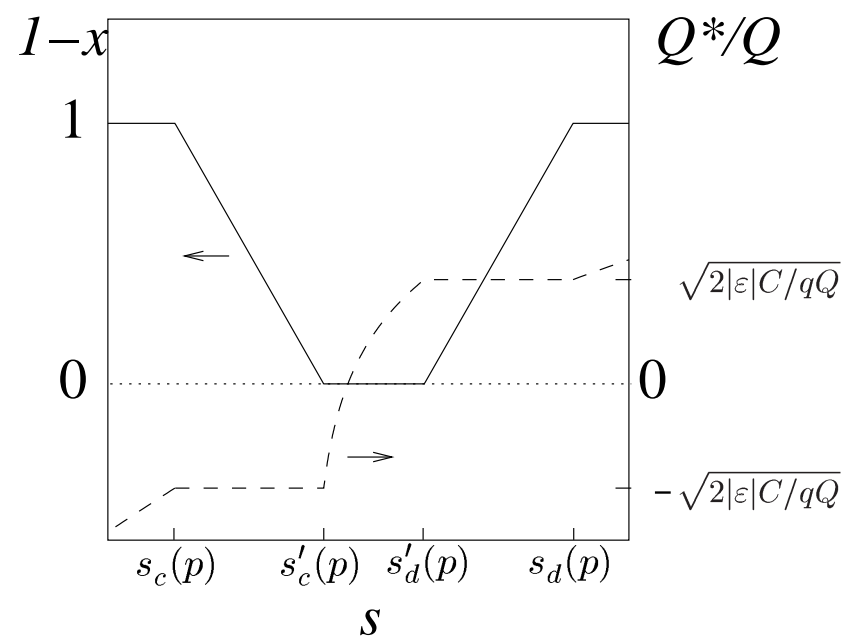

FIG. 7. The fraction $(1-x)$ of free DNA in solution and the charge $Q^{*}$ (in units of $Q$ ) of a free complex as a function of the concentration $s$ of spheres.

Let us now consider how the charge $Q^{*}$ of a free complexes varies from negative at $s<s_{0}(p)$ to positive at $s>s_{0}(p)$. When $s<s_{c}(p)$, Eq. (9) shows that $Q^{*}$ grows logarithmically with increasing $s$. However, in the first coexistent region, $s_{c}(p)<s<s_{c}^{\prime}(p)$, the net charge of the complex $Q^{*}$ equal $Q_{c}^{*}<0$ and stays constant. In the second coexistence region, $s_{d}^{\prime}(p)<s<s_{d}(p)$, the net charge is once more constant and but equal $Q_{d}^{*}=-Q_{c}^{*}>0$. When $s>s_{d}(p)$, this net charge increases again logarithmically with $s$. In Fig. 7, this net charge is plotted by the dashed line. In the region $s_{c}^{\prime}(p)<s<s_{d}^{\prime}(p)$, because the condensate consumes all the free complexes, we have to go beyond Eqs. (9) and (10) to find $Q^{*}$. Namely, in this interval we should take into account translational entropy of free complexes. This gives a new equation which replaces Eq. (10):

$$
N_{i}|\varepsilon|=\left(\frac{N}{N_{i}}-1\right)^{2} \frac{Q^{2}}{2 C}-k_{B} T \ln \left[(1-x) p V_{0}\right]
$$

where $(1-x) p$ is the concentration of free complexes in equilibrium with the condensate and $V_{0}$ is the normalizing volume for the complexes.

Since we are interested in the variation of the net charge $Q^{*}=Q\left(N / N_{i}-1\right)$ with respect to $s$, let us eliminate $x$ from the two Eqs. (9) and (27). This gives: 


$$
\begin{array}{r}
s-p N_{i}=s_{0} \exp \frac{q Q^{*}}{k_{B} T C}+\frac{N_{i} v_{0}}{V_{0}} s_{0}\left(\frac{N}{N_{i}}-1\right) \times \\
\exp \left(\frac{\left|\mu_{c}\right|-N_{i}|\varepsilon|}{k_{B} T}\right) \exp \frac{Q^{* 2}}{2 C k_{B} T} .
\end{array}
$$

For large $N_{i}$ (long DNA), the second term in the above equation is exponentially small compared to the first term, thus one has:

$$
Q^{*}=\frac{k_{B} T C}{q} \ln \left(1+\frac{s-s_{0}(p)}{s_{0}}\right) .
$$

Using Eqs. (13) and (15) one can verify that $Q^{*}$ matches $Q_{c}^{*}$ and $Q_{d}^{*}$ at $s=s_{c}(p)$ and $s=s_{d}(p)$ respectively.

It is interesting to note that the region where the complexes inverted charge is relatively narrow. Indeed, the slope at which $Q^{*}$ changes sign from negative to positive at $s_{0}(p)$ is

$$
\frac{\partial Q^{*}}{\partial s}=\frac{Q}{s_{0}} \frac{k_{B} T 2 C}{q Q} .
$$

Thus the characteristic width $\delta s=2 Q_{d}^{*} /\left(\partial Q^{*} / \partial s\right)$ over which charge inversion happens is exponentially smaller than $s_{d}-s_{c}$.

\section{MICROSCOPIC THEORY}

In the previous sections, we presented a description of the correlation-driven reentrant condensation and obtained an universal phase diagram based on two phenomenological parameters $\varepsilon$ and $s_{0}$. These two parameters depend on microscopic properties of the specific system considered.

In this section we use a microscopic theory of the beads-on-string structure (Fig. 11) for calculation of $\varepsilon$, $s_{0}$ and their dependence on monovalent salt concentration. We consider large spheres and, therefore, effectively work in the "neck" of the phase diagram (large $p$ case).

First, for simplicity, we start from the theory for polyelectrolyte with linear charge density $\eta \leq \eta_{c}=k_{B} T / e$. The next section considers highly charge polyelectrolyte and the Onsager-Manning condensation which renormalizes the net charge of DNA resulting in a shift of the isoelectric line.

Because the DNA coil winding around a sphere almost neutralizes the sphere charge, the correlation chemical potential $\mu_{c}$ is essentially the self energy of a bare free sphere in solution which is almost totally eliminated in the complext. Thus one has

$$
\mu_{c}= \begin{cases}-q^{2} / 2 D R & r_{s}>R \\ -q^{2} r_{s} / 2 D R^{2} & r_{s}<R\end{cases}
$$

The parameter $s_{0}$ can be easily found with the help of Eqs. (8) and (31). One sees that, at large $r_{s}$, the concentration $s_{0}$ is almost constant but when $r_{s}$ decreases below $R, s_{0}$ increases exponentially with $r_{s}$.
To calculate the correlation attraction energy $\varepsilon$, let us use Fig. 2. When two spheres touch each other, the density of the solenoid at the touching region doubles. This leads to a gain in the correlation energy of DNA turns (these correlations develop at distances of the order of $A$ and should not be confused with the correlation between spheres which determines $\mu_{c}$ and develops at distance of the order of $R$ ).

The correlation energy per unit length of the DNA can be estimated as the interaction energy of the DNA segment with its stripe of background (sphere) positive charge. Thus it is $-\eta^{2} \ln (R / A) / D$ for $r_{s}>R$ and $-\eta^{2} \ln \left(r_{s} / A\right) / D$ for $R>r_{s}>A$. Correspondingly, when the density doubles ( $A$ halves), the gain in the correlation energy per unit length is $-\eta^{2} / D$ for $r_{s}>A$. Similar effect takes place at $r_{s}<A$. In this case, each DNA interacts with the stripe of the width of $r_{s}$ of the sphere surface with energy $-q \eta r_{s} / 2 D R^{2}$. When two spheres touch each other, it interacts with the stripe on the other sphere as well, doubling the correlation energy. Thus in this case, the correlation energy gain is $-q \eta r_{s} / 2 D R^{2}$. Simple geometrical calculation shows that the total length of DNA in the touching region (the region surrounded by broken line in Fig. 2) is $R$. Therefore,

$$
\varepsilon= \begin{cases}-R \eta^{2} / D & r_{s}>A, \\ -q \eta r_{s} / 2 D R & r_{s}<A .\end{cases}
$$

Using Eqs. (31) and (32), we are now in a position to discuss in detail the change of the phase diagram with varying monovalent salt concentration (varying $r_{s}$ ). Let us consider the following three regimes: $r_{s}>R$, $R>r_{s}>A$ and $r_{s}<A$

In the first regime, $r_{s}>R$, the parameters $s_{0}$ and $\varepsilon$ remain constant. The capacitance $C$ of a complex has the form:

$$
C=D L / 2 \ln \left(r_{s} / R\right), \quad r_{s}>R,
$$

where in Eq. (3) the average distance $l$ between spheres has been replaced by $2 R$, because, near the condensation region, complexes are almost neutral and spheres almost touch each other. Therefore, Eq. (33) and (10) show that, in the coexistence regions, the complex charge $Q\left(N_{c, d} / N_{i}-1\right)$ increases logarithmically with decreasing $r_{s}$. Eq. (25) then shows that the relative width of the condensation region increases slowly in this regime:

$$
\frac{\Delta s(p)}{s_{0}(p)} \sim \frac{2 R \eta}{q} \sqrt{\frac{1}{\ln \left(r_{s} / R\right)}} \ll 1 .
$$

Here, Eq. (3) and the equality $Q / L=q / R$ were used. Recall that we assume the number of turns, $m$, of DNA around a sphere is large. Therefore $R \eta / q \simeq m^{-1} \ll 1$. Decreasing $r_{s}$, however, leads to the contraction of the condensation region in the limit $p \rightarrow 0$. Indeed, Eq. (13) shows that at constant $\varepsilon$, the concentration $s_{c}$ increases and the concentration $s_{d}$ decreases. Thus, overall, the 
condensation region decreases linearly with decreasing $r_{s}$ at small $p$ and increases logarithmically at large $p$.

In the second regime, $R>r_{s}>A$, The concentration $s_{0}$ increases as $\exp \left(-r_{s} / R\right)$ while $\varepsilon$ remains constant. The phase diagram moves upward with $s_{0}$. Since $r_{s}<R$, the capacitance $C$ is

$$
C=D L R / 2 r_{s}, \quad r_{s} \ll R .
$$

Thus, the relative width of the condensation region increases faster than Eq. (34):

$$
\frac{\Delta s(p)}{s_{0}(p)} \sim \frac{2 R \eta}{q} \sqrt{\frac{R}{r_{s}}} .
$$

(Notice that Eq. (36) matches Eq. (34) at $r_{s} \sim R$ ). At the same time, at small $p$, the condensation region shrinks faster $s_{c, d} \propto \exp \left(\mp r_{s} / R\right)$. Thus in this regime, the change in shape the phase diagram continues the trend in the first regime but with a faster pace.

In the third regime, $r_{s}<A$, the energy $\varepsilon$ also starts to decrease linearly with $r_{s}$. Eqs. (10) and (13) therefore lead to the saturation in the change in $s_{c, d}$ and $N_{c, d}$. The relative width of the condensation region in the "neck" saturates at:

$$
\frac{\Delta s(p)}{s_{0}(p)} \sim \sqrt{\frac{2 R \eta}{q}} .
$$

(Notice that, because $A \sim R^{2} \eta / q$, Eqs. (36) and (37) match each other at $r_{s} \sim A$.) On the other hand, the concentration $s_{0}$ continues to increase exponentially with decreasing $r_{s}$. One can say that in this region, the main effect of increasing salt concentration is the growth of the "body" of the phase diagram Fig. A at the expense of the "neck".

The evolution of the width of the "neck" as function of $r_{s}$ is shown on Fig. 8.

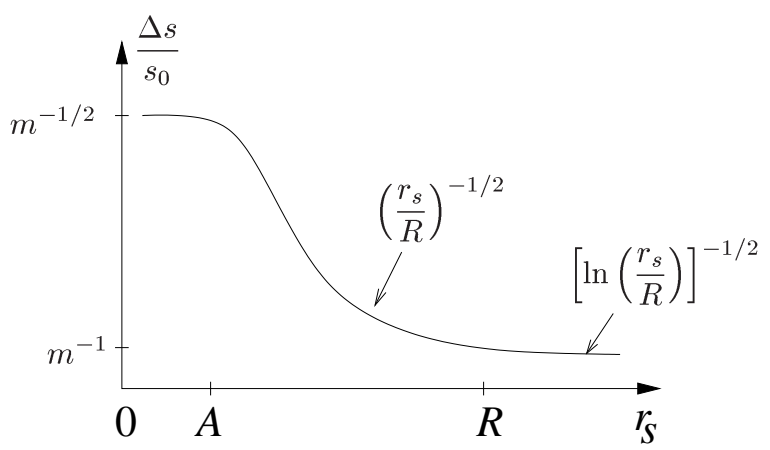

FIG. 8. The relative width of the condensation region of DNA-spheres complexes as a function of the screening length. $m=q / \eta R \gg 1$ is the number of turns of DNA neutralizing a sphere.

Concluding this section, we would like to justify quantitatively the assumption of Eq. (7), which leads to conclusion that the charge of complexes is small near condensation region. Using Eq. (32) for $\varepsilon$ and Eqs. (33) and Eq. (35) for the complex capacitance $C$ one gets:

$$
\frac{|\varepsilon| C}{q Q}= \begin{cases}(R \eta / q)^{2} / \ln \left(r_{s} / R\right) & r_{s}>R, \\ (R \eta / q)^{2} R / r_{s} & R>r_{s}>A \simeq R^{2} \eta / q, \\ (R \eta / q) & A>r_{s} .\end{cases}
$$

One sees that in all cases, the ratio $|\varepsilon C / q Q|$ is at most $R \eta / q=m^{-1}$ where $m$ is the number of turns of DNA coil on a sphere which we assume to be large. Thus the inequality (7) is justified.

\section{ONSAGER-MANNING CONDENSATION}

The theory presented so far is applicable to the complexation of polyelectrolyte with linear charge density which does not exceed the so called Onsager-Manning critical charge density $\eta_{c}=D k_{B} T / e$. DNA helix is, however, a highly charged polyelectrolyte with the bare negative charge density $-\eta \simeq-4.2 \eta_{c}$. Condensation of positive monovalent ions on DNA, therefore, must be taken into account. This section deals with the problem of counterion condensation.

For a free DNA helix, the Onsager-Manning condensation results in the net charge $-\eta_{c}$. However, the net linear charge density of DNA winding around a charged sphere, $-\eta^{*}$, may differ from $-\eta_{c}$ due to the repulsion of counterions from the positive charge of the sphere. This phenomenon is called counterion release 19 . We show below that, for DNA coil which neutralizes a sphere, $\eta^{*}$ is given by Eq. (11), so that $\eta \geq \eta^{*} \geq \eta_{c}$. Similar to $L$ and $l$, the net charge density of DNA, $\eta^{*}$, can be considered as independent on $s, p$ and $N$, if one is interested only in the reentrant condensation domain of the phase diagram where the charge of complexes are small. Therefore, the theory used in previous section remains valid provided one replaces $Q$ by $Q^{*}=Q \eta^{*} / \eta, N_{i}$ by $Q^{*} / q$ and $s_{i}$ by $p Q^{*} / q$.

Remarkably, because the relative width of the condensation domain studied in Sec. III is small with or without screening, when Onsager-Manning condensation is taken into account the main effect of screening in the phase diagram is the decrease of $Q$ and, correspondingly, the isoelectric concentration $s_{i}$. The whole right part of diagram of Fig. 4 shifts in the direction of lower $s$ together with the renormalized $s_{i}$, as it is shown in Fig. 6. To understand the implications of such shift, let us consider a solution 1 , which is represented by the point $(s, p)$ below the curve $s_{c}(p)$ at very large $r_{s}$, when $\eta^{*}$ is equal to the bare DNA charge density $\eta$. If we add more monovalent salt and reduce $r_{s}$, both curves $s_{c}(p)$ and $s_{d}(p)$ move down following the move of the isoelectric line. As the result, our point 1 can be found inside the domain where complexes aggregate. Thus condensation of negative (underscreened) complexes of DNA and spheres can be induced by addition of salt. 
On the other hand, if we start from the solution represented by the point 2, which at large $r_{s}$ is above the decondensation line $s_{d}(p)$, the result is completely different. In this case, addition of salt can not condense DNA-spheres complexes. The origin of this asymmetry is that we are considering large enough spheres with surface charge density smaller than that of DNA helix, so that only monovalent cations experience the OnsagerManning condensation.

It should be noted here that all the discussion above about the change in the isoelectric line is relevant for the "neck" of the phase diagram only. The expansion of the "body" toward higher $p$ and $s$ with decreasing $r_{s}$ at $r_{s}<R$ mentioned in the previous section is not affected by the Onsager-Manning condensation.

Let us now derive Eq. (11) for the net linear charge density of the DNA due to Onsager-Manning condensation. Let us denote by $c_{1}$ the concentration of monovalent salt in the solution and by $c_{s}$ the concentration of monovalent ions condensed on the DNA surface. As we know, for a free DNA in solution, counterions condense on DNA making its net charge $-\eta_{c}$. The balance of the chemical potential of counterions in solution and those condensed on the DNA helices, then reads

$$
k_{B} T \ln \frac{c_{s}}{c_{1}}=\frac{2 e \eta_{c}}{D} \ln \frac{r_{s}}{a},
$$

where the left hand side is the entropy lost and the right hand side is the energy gained when a counterion condenses on the DNA surface, $a$ is the radius of the DNA double helix (not to be confused with the radius $l$ of the complex, which, for almost neutral complexes where spheres almost touch each other, is roughly the radius of one sphere, $l \simeq R \gg a$ ).

In the DNA-sphere complex, the positive charge of the sphere reduces the amount of the counterions condensed on the DNA making the net linear charge of DNA equal $-\eta^{*}$, instead of $-\eta_{c}$. The equilibrium condition for the monovalent counterions now reads

$$
k_{B} T \ln \frac{c_{s}}{c_{1}}=\frac{2 e \eta^{*}}{D} \ln \frac{A / 2 \pi}{a}-e \psi(0) .
$$

Here $A / 2 \pi=2 R^{2} \eta^{*} / Q$ plays the role of the local screening length at the DNA surface ( $A$ is the distance between subsequent turns of DNA around a sphere (see Fig. 11)). If $A / 2 \pi \gg r_{s}$ then $A / 2 \pi$ should be replaced by $r_{s}$. The average electrostatic potential at the surface of a DNAsphere complex is $\psi(0)=\left[2 Q^{*}\left(N / N_{i}-1\right) / D L\right] \ln \left(r_{s} / R\right)$. (Here and below, bearing in mind that we are working in the vicinity of isoelectric point we replaced $l$ by $R$ ).

Combining Eqs. (39) and (40), one gets

$$
\eta_{c} \ln \frac{r_{s}}{a}=\eta^{*} \ln \frac{A}{2 \pi a}-\frac{Q^{*}}{L}\left(\frac{N}{N_{i}}-1\right) \ln \frac{r_{s}}{R}
$$

Near the condensation domain, the charge of a complex $\left(N / N_{i}-1\right)$ is very small and the second term in the right hand side in Eq. 41) is negligible. (Indeed, according to eq. (9), $Q^{*}\left(N / N_{i}-1\right) / L \sim k_{B} T D / q \ll k_{B} T D / e=\eta_{c}$, and because $R \gg a$, the second term is much smaller than $\left.\eta_{c} \ln \left(r_{s} / a\right)\right)$. Using $A / 2 \pi=2 \eta^{*} R^{2} / Q$ and substituting $\eta^{*}$ by $\eta_{c}$ in $\ln (A / 2 \pi a)$, one arrives at $N$-independent net linear charge density for DNA given by Eq. (11). This net charge decreases with decreasing $r_{s}$ (increasing salt concentration). When $r_{s}$ reaches $A / 2 \pi$, the net charge density $\eta^{*}$ reaches $\eta_{c}$. When $r_{s}$ is even smaller, $A / 2 \pi$ is replaced by $r_{s}$ and $\eta^{*}$ saturates at the value of $\eta_{c}$.

The above derivation of $\eta^{*}$ is similar to that of Ref. 20 where the problem of adsorption of DNA (with its Onsager-Manning condensed counterions) on a charged surface was considered. However, the obtained net charge (Eq. (11)) is different with that of Eq. (7) of Ref. 20. This is because Ref. 20 considers the case when DNA strongly overcharges the surface, while this paper concerns with almost neutral DNA-spheres complexes.

In the final paragraph of this section, we would like to discuss the condensation of negative monovalent ions (coions of DNA) on the spheres. This happens if the surface charge density of the sphere is large enough and the screening radius is not very large 28 . Near the condensation domain, however, DNA coil almost neutralizes a sphere eliminating its Coulomb potential at distances larger than $A / 2 \pi$. Therefore, most of coions are released from the sphere. There is still a small amount of them condensed in the middle between two DNA turns. However, the total charge of these coions is much smaller than the total charge of DNA counterions condensed on the DNA because, for $A \gg a$, DNA has a much larger bare surface charge density. Thus these coions are not important in our calculation of the isoelectric point. This also means that our calculation of $\varepsilon$ is valid. On the other hand, $\mu_{c}$ can be modified when sphere condensation is taken into account because the self energy of the sphere in this case is smaller than that given by Eq. (31). However, $\mu_{c}$ affects only $s_{0}$. For large spheres where Coulomb interaction is much larger than $k_{B} T$, we always are in the "neck" of the phase diagram and $s_{0}$ is irrelevant. Therefore, the effect of condensation of DNA coions on spheres is small.

\section{OTHER APPLICATION OF PHENOMENOLOGICAL THEORY OF REENTRANT CONDENSATION}

In Sec. II, we presented a theory of the phase diagram for complexation of a long DNA helix with oppositely charged spheres. It shows that correlation induced charge inversion leads to reentrant condensation. The phase diagram is described by two parameters $\varepsilon$ and $s_{0}$. Beside these two parameters, however, the phenomenological theory does not use any specific information about the structural and chemical properties of the DNA. Therefore, this theory is generic and can be used to describe 
a broad range of systems experiencing reentrant condensation. In this section, we would like to discuss briefly a number of other systems of large opposite charges which can be described by the same phase diagram of Fig. 4 .

Spheres can be replaced by particles of any shape which we for brevity call $Z$-ions. There is no need for them to be rigid. For example, they can be star micelles or just oppositely charged shorter polyelectrolytes (PE), more flexible than DNA, so that DNA can be considered rigid. Still they will complex with DNA, for example, winding around it, repel each other on DNA and form a kind of necklace. Phenomenological parameters $s_{0}, \varepsilon$ can still be introduced (but, of course, microscopic calculation of these parameters is different from that of Sec. III) and the phase diagram should look similar to Fig. A or Fig. 5.

Let us discuss the limit when the $Z$-ion size is smaller than double helix diameter so that they form two dimensional correlated liquid on the surface of DNA. A well studied example of this limit is the reentrant condensation of DNA in the presence of spermine already mentioned in Introduction. Microscopically, it is different from the beads-on-string systems, because in this case DNA helices can be considered as rigid cylinders. Condensation of DNA, of course, requires attraction between like-charged cylinders. This attraction is related to the fact that $Z$-ions, at the surface of DNA cylinders, strongly repel each other and form a two-dimensional strongly correlated liquid. At the place of the contact where the correlated liquids of two touching DNA merge, the two-dimensional concentration of $Z$-ions doubles. This leads to an energy gain 2125 because the correlation energy of a strongly correlated liquid per $Z$-ion is known to be negative and increases in absolute value with increasing $Z$-ion concentration. In other words, two DNA helices with strongly correlated liquid of $Z$-ions on their surfaces experience a correlation-induced short range attraction.

It was predicted2627 that in a very weak DNA solution, the net charge of a DNA helix inverts sign at a critical concentration $s=s_{0}$. It has the meaning of concentration of $Z$-ions in solution which is in equilibrium with the strongly correlated liquid of $Z$-ions at the surface of DNA (concentration of "saturated vapour" above strongly correlated liquid ). Physics of this inversion of charge is also related to correlations: when a new $Z$-ion approaches already neutralized DNA it forms an image in strongly correlated liquid, which attracts it.

Combining correlation induced charge inversion and short range attraction, Ref. 17 offered an explanation for the origin of the reentrant transition at small concentration of DNA. At $s<s_{0}$ charges of two helices are negative. Their absolute values decrease with increasing $s$ until at $s=s_{c}$, where Coulomb repulsion looses to the correlation attraction and DNA condenses in bundles. At $s>s_{0}$ the net charge of DNA becomes positive and grows with increasing $s$. At $s=s_{d}$, the Coulomb repulsion wins over correlation attraction and DNA bundles dissolve. For $p \rightarrow 0$ Eqs. (12), (13) were derived already in Ref. 17. What is done in phenomenological theory of Sec. II can be considered as extension of the theory of Ref. 17 to arbitrary $p$.

Applicability of the phase diagram of Fig. 1 1 of course, is not limited to DNA. For all the broad spectrum of $Z$ ions, long DNA helices, in turn, can be replaced by other long, strongly charged PE. Experiment on complexation in mixture of micelles and oppositely charged PEE is a good example. In this experiment the total charge of micelles was varied by changing the concentration of the cationic lipid in the solution. At some critical point the electrophoretic mobility of complexes changes sign. In agreement with the above theory measurements of dynamic light scattering and turbidity (coefficient of light scattering) show that complexes condense in bundles in the same vicinity of the point where mobility crosses over between two almost constant positive and negative values.

An interesting new case is that of a weakly charged (one charge per $1 / f \gg 1$ monomers) flexible PE. Let us consider its complexation with small strongly charged spheres. In this case, when PE winds around a sphere, the entropy of PE chain prevents its collapse to the surface of the sphere. As a result PE builds around the sphere layers of blobs with changing size, similar to the one discussed in Ref. 29. The effective radius of a small sphere can be renormalized to a larger value. The rest of theory of complexation is similar to Ref. 8 and this paper so that our phase diagram works in this case, as well.

Complexation of a long PE with much shorter oppositely charged $\mathrm{PE}$ in a water solution is important class of such problems. Let us assume that the long PE is flexible while the shorter one is rigid and stronger charged. Then the long one sequentially wraps around molecules of the short one so that rods-on-a-string system resembling Fig. 1 is formed. Then the only change to be made in the theory of Ref. 8 and this paper is to replace self energy of a charged sphere by self energy of a charged rod. The case when the short polymer is flexible (but still stronger charged) on the first glance seems to be more complicated. However, away from isoelectric point short PE is either underscreened or overscreened by the wrapping long one, so that it has the rode-like shape. Similarly the whole complex is charged, stretched and always resembles Fig. 1. Finally, if both PE are weekly charged but even the short one is long enough, so that its charge is large, they still form a complex resembling Fig. 11 with some renormalizations of geometrical parameters related to a hierarchy of blobs.

A long DNA helices or long PE molecules can be also replaced by any large macroions, for example, a large colloidal particle, which adsorbs smaller $Z$-ions. With appropriate correction for the expression of the capacitance $C$ of the complex which, in this case, has spherical instead of cylindrical shape, our theory is applicable as well. An interesting practical example of such system is solution of positive (latex) spheres with short DNA 
oligomers with, say, \&or 16 bases, which are adsorbed at the surface of spheres 3 . Modified DNA can be delivered by such colloids as a drug. Therefore question of stability of such solutions at a given concentration of DNA is very important.

It is interesting that in this case latex spheres play the role of long DNA helix in the theory of our paper, while short DNA oligomers play the role of spheres or $Z$-ions. Indeed, short DNA oligomers form two-dimensional correlated liquid on the surface of colloid and can overscreen it. Simultaneously this liquid provides attraction of almost neutralized colloids leading to reentrant condensation around isoelectric point. Reentrant condensation and change of the sign of electrophoretic mobility in these systems were carefully studied experimentally 3 , the results seem to agree with our theory. Electrophoretic mobility of free complexes changes sign in the very narrow vicinity of isoelectric point where solution is unstable and easily coagulates.

Condensation of colloids with multivalent $Z$-ions was recently observed in Monte-Carlo experiments 30 although the concentration of $Z$-ions are not large enough to observe resolubilization.

Another example of application of our phase diagram to almost spherical macroions is the solution of nucleosomes with spermine 16 . In the nucleosome the positive histone octamer (charge $\sim 160 e$ ) is strongly overcharged by DNA with charge -292e. Adsorption of spermine cations on the surface of nucleosomes leads to nucleosome attraction and aggregation. Phase diagram of this system looks like the body part of phase diagram of Fig. 4. Electrophoretic mobility was measured for aggregates of nucleosomes. With increasing concentration of spermine it changes sign from negative to positive roughly in the middle between condensation and decondensation curves $s_{c}(p)$ and $s_{c}(p)$ (in logarithmic scale).

\section{LIMITATIONS OF REENTRANT CONDENSATION.}

In the previous section, we saw that the combination of reentrant condensation and charge inversion is a very general phenomenon. Now we would like to understand whether this phenomenon takes place for any strongly charged and strongly interacting electrolyte. We start from a theoretical example of a system, which does not show this phenomenon. This will help us to formulate limitations for reentrant condensation and charge inversion.

Let us consider complexation of an absolutely rigid cylinder like negative $\mathrm{PE}$ with small and strongly charged $Z$-ions24. Let us assume that linear charge density of PE is $\eta$, radius of the cylinder is $a$ and it is larger than radius of $Z$-ion. We assume also that $\mathrm{PE}$ is not very strongly charged so that $Z / a \eta \gg 1$. In this case, isolated complexes of a cylinder with adsorbed $Z$-ions resemble onedimensional Wigner crystal. Two such one-dimensional
Wigner crystals strongly attract each other. The deep minimum of Coulomb energy is always provided by the condensed state, where cylinders form densely packed background on the top of which $Z$-ions form a threedimensional strongly correlated liquid with average distance between $Z$-ions much larger than $a$ (they actually sit in pores of the background). The binding energy of such strongly correlated liquid is much larger than for free one-dimensional complexes. Therefore, at large concentrations of $\mathrm{PE}$ and $Z$-ions, this system is always in condensed state and there is no way to see reentrant condensation.

This (not very realistic) example emphasizes nontrivial nature of reentrant condensation and charge inversion in the systems of DNA with large spheres or with spermine (and other similar systems discussed above). The destruction of aggregates by Coulomb repulsion of complexes at small distance from neutrality line (reentrant condensation) is possible only if the condensate binding energy per $Z$-ion, $\varepsilon$, is smaller than the Coulomb interaction of a $Z$-ion with macroion, $q Q / C$. Inequality $\varepsilon /(q Q / C) \ll 1$ was used in Sec. II to simplify calculations. If it is violated, the net charge $Q_{c, d}^{*}$ is not small, the neck widens and can occupy the whole plane at large $s$ and $p$ eliminating condensation and decondensation transitions. Of course, the requirement that interaction between $Z$-ion and DNA is large $\left(\left|\mu_{c} \gg k_{B} T\right|\right)$, so that charge inversion can happen, is also necessary for an observation of reentrant condensation.

Small values of $\varepsilon$ are realized for DNA with large spheres or with spermine because in both cases charges which form complexes very strongly screen each other. For DNA with large spheres charge of a sphere is well screened by coiling DNA so that spheres stick together only at the place they touch each other (Fig. 2) and the energy $\varepsilon$ is very small. In the case of DNA with spermine, $Z$-ions so strongly screen DNA that attraction is developed only in the small area where two DNA touch and the energy $\varepsilon$ is relatively small.

Between systems of DNA with large spheres and DNA with spermine there are many others with intermediate size and charge of spheres. One can easily imagine an intermediate system, where on one side spheres are not large and strongly charged enough that DNA behaves as a flexible PE, and on the other side they are strongly enough charged so that they form something like onedimensional Wigner crystal on its surface. In such a case, mutual screening of DNA and spheres is not as complete as in the case of DNA with large spheres or with spermine (see Fig. 9 or Ref. 32). Therefore, inequality $|\varepsilon| /(q Q / C) \ll 1$ can be violated and reentrant condensation may not materialize. 


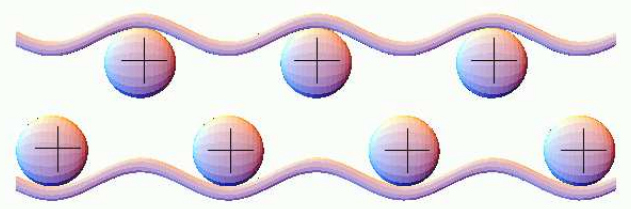

FIG. 9. Complexation of DNA and spheres with intermediate value of charge and size. Screening is not complete as in the case of large spheres or multivalent ions.

Let us give an example of a different, well-studied system where this happens. Consider a solution cationic lipid membranes and long double helix DNA. Each membrane, in principle, may complex with many DNA rods which lie on it equidistantly parallel to each other, forming a kind of one-dimensional Wigner crystal. (In such a complex DNA plays the role of $Z$-ion) These complexes may be undercharged and overcharged. They can also condense in three-dimensional aggregates, where layers of equidistantly positioned parallel DNA rods alternate with membranes 11 . Such aggregates have potential applications in gene therapy as non-viral gene carriers. In spite of liquid nature of membranes, which permits some redistribution of lipid charge inside membrane to screen DNA, attraction between different complexes in this crystal is so strong that situation for a reentrant transition in this case is marginal $(|\varepsilon| C / q Q \sim 1)$. It seems from experimental data that ingeed aggregates exist even far from the isoelectric point 31 .

For the colloidal spheres covered by short DNA mentioned in Sec. V, binding is related to the small area of contact and energy $\varepsilon$ is small again. Let us, instead of short DNA, consider small positive colloidal particles. If their size and charge are much smaller than that of larger negative ones we still get reentrant condensation like with the short DNA. But what happens when their charges and sizes become comparable is not obvious. Therefore it is interesting to consider a simpler case of a solution of positive and negative spherical macroions with same absolute values of charges, $q$, same radius, $R$ and with concentrations $c_{+}$and $c_{-}$respectively. Let us talk about large enough $c_{+}$and $c_{-}$, when Coulomb energy of interaction of two touching spheres, $E=q^{2} / 2 D R$, completely dominates the entropy. It is obvious then that at $c_{+}=c_{-}$such system tends to self assemble into NaCl-like crystal and gain the Madelung energy $-1.74 E$ per negative sphere. Away from the isoelectric point situation is less trivial. Let us consider for simplicity a solution with $c_{+}=2 c_{-}$(neutrality is provided by monovalent negative ions). In this case one has to compare energies of two states. The first one consist of NaCl-like crystal consuming concentrations $c_{-}$of both positive and negative spheres. The rest $c_{-}$positive spheres are free. Such state has the energy $-1.74 E c_{-}$per unit volume of solution. The second state consists only of $c_{-}$triplets, in which two positive spheres are attached on opposite sides to a negative one. Energy of such a complex is $-1.5 E$.
We see that second state looses to the first. It can be shown that at any $c_{+} / c_{-}$, the largest possible fraction of spheres always self assembles in neutral NaCl-like crystal. Thus we are coming to conclusion that triplets (which are positive at $c_{+}>c_{-}$and negative at $c_{+}<c_{-}$) do not form. Triplets can be considered as analogs of free and charged DNA-spheres necklaces. So in this case, neutral condensed state always dominates and there is no reentrant condensation at all.

\section{CONCLUSION}

In this paper, we presented a phenomenological theory of reentrant condensation. The theory is applicable not only to the solution of DNA and spheres but also to a much broader range of systems.

Here, we would like to discuss possible implications of our theory for the natural chromatin assuming that the beads-on-a-string structure of $10 \mathrm{~nm}$ chromatin fiber is indeed determined by electrostatic interactions. In this case, the finite distance between nucleosomes tells us that the whole $10 \mathrm{~nm}$ fiber is charged. Electrophoretic experiment 33 show that in low salt conditions, the net charge of $10 \mathrm{~nm}$ fiber is negative. (By the net charge we mean bare charges of DNA and histones plus charges which are Onsager-Manning condensed on them and, therefore, are bound to the fiber with the energy larger than $\left.k_{B} T\right)$. An additional argument for the negative sign of the charge is that the increasing salt concentration condenses it into the $30 \mathrm{~nm}$ fiber. This means that $10 \mathrm{~nm}$ fiber obeys the scenario discussed in Sec. V for the point 1 of Fig. 6 .

Looking at the phase diagram of Fig. 6 we can ask whether one can increase concentrations of both octamers and salt much further so that that the point $(s, p)$ becomes higher than the decondensation curve $s_{d}(p)$ and instead of $30 \mathrm{~nm}$ fiber or higher order structures we can get a positive stable $10 \mathrm{~nm}$ fiber. This is not easy because free octamers decay into smaller histone dimers or tetramers. To keep equilibrium concentration of free octamers $s$ in solution at the necessary level, concentrations of histones should be large. In spite of this difficulty we suggest to try to create and study a positive $10 \mathrm{~nm}$ fiber.

\section{ACKNOWLEDGMENTS}

We are grateful to A. Yu. Grosberg, V. Lobaskin, E. Raspaud, I. Rouzina, U. Sivan and J. Widom for useful discussions. This work is supported by NSF DMR9985785 . 
${ }^{1}$ B. Alberts, D. Bray, J. Lewis, M. Raff, K. Roberts, and J. D. Watson, Molecular biology of the cell, Galland Publishing, New York (1994).

${ }^{2}$ E. Braun, Y. Eichen, U. Sivan and G. Ben-Yoseph, Nature, 391, 775 (1998).

${ }^{3}$ H. W. Walker, and S. B. Grant, Colloids and Surfaces A, 119, 229 (1996).

${ }^{4}$ N. Götting, H. Fritz, M. Maier, J. von Stamm, T. Schoofs, and E. Bayer, Colloid Polym. Sci. 277, 145 (1999).

${ }^{5}$ Y. Wang, K. Kimura, Q. Huang, P. L. Dubin, W. Jaeger, Macromolecules, 32 (21), 7128 (1999).

${ }^{6}$ Y. Wang, K. Kimura, and P. Dubin, Macromolecules 33, 3324 (2000); Y. Li, P. Dubin, H. A. Havel, S. L. Edwards, and H. Dautzenberg, Langmuir 11, 2486 (1995).

${ }^{7}$ H. M. Evans, A. Ahmad, T. Pfohl, A. Martin, C. R. Safinya Bull. APS, 46391 (2001).

${ }^{8}$ T. T. Nguyen, and B. I. Shklovskii, J. Chem. Phys. 114, 5905 (2001).

${ }^{9}$ G. S. Manning, J. Chem. Phys. 51, 924 (1969).

${ }^{10}$ M. G. Bungenberg de Jong, in Colloid science, Kruyt H. R. ed., Elsevier, New York (1990), Vol. 2. Note that the signs plus and minus in this reference are used only for the charge of aggregates (coacervate) droplets while free complexes are not discussed at all.

${ }^{11}$ V. A. Bloomfield, Curr. Opinion Struct. Biol.6, 334 (1996); Biopolymers. 44, 269 (1998).

12 M. Saminathan, T. Antony, A. Shirahata, L. Sigal, T. Thomas and T. J. Thomas, Biochemistry. 38, 3821 (1999).

13 J. Pelta, D. Durand, J. Doucet and F. Livolant, Biophysical Journal. 71, 48 (1996).

14 J. Pelta, F. Livolant and J.-L. Sikorav, Journal of Biological Chemistry. 271, 5656 (1996).

${ }^{15}$ E. Raspaud, M. Olvera de la Cruz, J.-L. Sikorav and F. Livolant, Biophysical Journal. 74, 3811998.

${ }^{16}$ E. Raspaud, I. Chaperon, A. Leforestier, and F. Livolant,
Biophysics J. 77, 1547 (1999).

17 T. T. Nguyen, I. Rouzina, and B. I. Shklovskii, J. Chem. Phys. 112, 2562 (2000).

${ }^{18}$ K. Keren, Y. Soen, G. Ben Yoseph, R. Yechieli, E. Braun, U. Sivan, and Y. Talmon, 2001, Private communication.

${ }^{19}$ S. Y. Park, R. F. Bruisma, and W. M. Gelbart, Europhys. Lett. 46, 493 (1999).

20 T. T. Nguyen, A. Yu. Grosberg, B. I. Shklovskii, J. Chem. Phys. 113, 1110 (2000).

${ }^{21}$ I. Rouzina and V. A. Bloomfield, J. Phys. Chem. 100, 9977 (1996).

${ }^{22}$ N. Gronbech-Jensen, R. J. Mashl, R. F. Bruinsma, and W. M. Gelbart, Phys. Rev. Lett. 78, 2477 (1997).

${ }^{23}$ Y. Levin, J. J. Arenzon, and J. F. Stilck, Phys. Rev. Lett. 83, 2680 (1999).

${ }^{24}$ B. I. Shklovskii, Phys. Rev. Lett. 82, 3268 (1999).

${ }^{25}$ A. G. Moreira, R. R. Netz, cond-mat/0009377

${ }^{26}$ V. I. Perel and B. I. Shklovskii, Physica A 274, 446 (1999).

${ }^{27}$ B. I. Shklovskii, Phys. Rev. E 60, 5802 (1999).

${ }^{28}$ M. Guerom, G. Weisbuch, Biopolimers, 19, 353 (1980); S. Alexander, P. M. Chaikin, P. Grant, G. J. Morales, P. Pincus, and D. Hone, J. Chem. Phys. 80, 5776 (1984); S. A. Safran, P. A. Pincus, M. E. Cates, F. C. MacIntosh, J. Phys. (France) 51, 503 (1990).

29 A. V. Dobrynin, A. Deshkovski and M. Rubinstein, Macromolecules 34, 3421 (2001).

${ }^{30}$ P. Linse, and V. Lobaskin, Phys. Rev. Lett. 83, 4208 (1999); J. Chem. Phys. 112, 3917 (2000).

${ }^{31}$ I. Koltover, T. Salditt, and C. R. Safinya, Biophys. J. 77, 915 (1999).

${ }^{32}$ R. R. Netz, and J.-F. Joanny, Macromolecules 32, 9013 (1999); K. K. Kunze, and R. R. Netz, Phys. Rev. Lett. 85, 4389 (2000).

${ }^{33}$ T. M. Fletcher, O. Krishnan, P. Server, and J. C. Hansen, Biochemistry 33, 2226 (1994). 